\title{
Non-intrusive techniques of inspections during the pre-launch phase of space vehicle
}

\author{
Rajkumar Thirumalainambi ${ }^{a}$ and Jorge E. Bardina ${ }^{b}$ \\ aSAIC@NASA Ames Research Center, Mail Stop 269-2, Moffett Field, California, USA; \\ ${ }^{b}$ NASA Ames Research Center, Mail Stop 269-2, Moffett Field, California, USA
}

\begin{abstract}
This paper addresses a method of non-intrusive local inspection of surface and sub-surface conditions, interfaces, laminations and seals in both space vehicle and ground operations with an integrated suite of imaging sensors during pre-launch operations. It employs an advanced Raman spectrophotometer with additional spectrophotometers and lidar mounted on a flying robot to constantly monitor the space hardware as well as inner surface of the vehicle and ground operations hardware. This paper addresses a team of micro flying robots with necessary sensors and photometers to monitor the entire space vehicle internally and externally. The micro flying robots can reach altitude with least amount of energy, where astronauts have difficulty in reaching and monitoring the materials and subsurface faults. The micro flying robot has an embedded fault detection system which acts as an advisory system and in many cases micro flying robots act as a 'Supervisor' to fix the problems. As missions expand to a sustainable presence in the Moon, and extend for durations longer than one year in lunar outpost, the effectiveness of the instrumentation and hardware has to be revolutionized if NASA is to meet high levels of mission safety, reliability, and overall success. The micro flying robot uses contra-rotating propellers powered by an ultra-thin, ultrasonic motor with currently the world's highest power weight ratio, and is balanced in mid-air by means of the world's first stabilizing mechanism using a linear actuator. The essence of micromechatronics has been brought together in high-density mounting technology to minimize the size and weight. The robot can take suitable payloads of photometers, embedded chips for image analysis and micro pumps for sealing cracks or fixing other material problems. This paper also highlights advantages that this type of non-intrusive techniques offer over costly and monolithic traditional techniques.
\end{abstract}

Keywords: Micro Flying Robot, Ground Operations, Space Vehicle inspection, Health monitoring of space vehicle, Non-intrusive techniques of inspection

\section{INTRODUCTION}

During the 1960s, NASA proved that access to space is feasible for both manned and unmanned systems. During the 1970s, NASA demonstrated that important scientific exploration and applications missions could be conducted in orbit. During the beginning of this century, NASA launched twin MARS rover to study martian rocks, and weather. During the next decade, NASA is planning to establish a human habitats on the Moon and Mars. A cost effective approach for the development of manned habitats on planetary surfaces is the use of robotic precursor missions for such tasks as the deployment and servicing of power systems, construction of beaconed roadways, and site preparation and deployment of manned habitat modules. On January 14th, 2004, the President of the United States gave an historic speech at NASA Headquarters in Washington, DC: "Today I announce a new plan to explore space and extend a human presence across our solar system. We will begin the effort quickly, using existing programs and personnel. We'll make steady progress - one mission, one voyage, one landing at a time." After President's vision was established, NASA decided to build the "Crew Exploration Vehicle" or (CEV) now called "Project Constellation". NASA will utilize a spiral development model to create variants of the $\mathrm{CEV}$ which can travel to earth orbit, to lunar orbit, to help conduct lunar landings, to build extended duration habitats, and to destinations beyond, such as Mars, near-earth asteroids, and the outer planets. ${ }^{1}$

Send correspondence to Dr. Jorge E. Bardina. E-mail: Jorge.E.Bardina@nasa.gov 
The development of the CEV represents the most significant new research into manned spacecraft since the mid-1960s. It will inherit the legacy and most likely the shape of the Apollo Command Module, but will benefit from almost 40 years of technological advances. While the CEV hardware is still early in the development, some of the concepts that may be adopted have already begun to emerge. Overall, a modular design is being touted in order for new capabilities to be added and to allow future launch vehicle designs to be easily swapped out. Wingshaped designs have not been ruled out yet, but many observers of the project, including the very astronauts that will ride the vehicle, are strongly in favor of a capsule shaped vehicle due to its proven flightworthiness during the Mercury, Gemini, and Apollo programs. Aside from the crew module itself, other modules are being designed to enable extended stays in space. Included in a series of graphic mockups released by Boeing is an inflatable habitat module, which many have already compared to a past NASA effort to develop an inflatable habitat for ISS, and as part of an interplanetary vehicle for future voyages to Mars.

NASA claims that the enormous distances between Earth, Mars and beyond mean that the only viable source of power to CEV is Nuclear Power. In 2004 NASA changed the name of its Nuclear Systems Initiative to Project Prometheus. Prometheus is targeted at utilizing nuclear energy for space exploration in two ways: Nuclear Fission based propulsion systems (nuclear rockets) to propel spacecraft, and Radioisotope based generators to provide energy for the mission tasks. Given the enormous complexity of the CEV, the pre-launch inspections of CEV and its ancillary systems play a crucial role in the success of mission. Shuttle launches currently take months of preparation at the launch pad. Millions of events/items have to be checked till the last count down of launch phase of any given mission. Validating the functionalities of an advanced automation of ground controls, and propellant systems of CEV before launch operations requires interaction of multiple colonies of robots, and probes in micro, mini and macro level. In this paper, we address a micro flying robot which supports nonintrusive techniques to analyze materials, and other surveillance for the CEV and extendability of micro flying robots to other areas of future space flight.

\section{WHY A NON-INTRUSIVE INSPECTION?}

The Columbia Accident Investigation Board issued a major recommendation to NASA. Prior to return to flight, NASA should develop and implement a "comprehensive inspection plan" to determine the structural integrity of all Reinforced Carbon-Carbon (RCC) system components. This inspection plan should take advantage of advanced non-destructive inspection technology. This recommendation was issued in request to the board's finding that current inspection techniques are not adequate to assess structural integrity of the RCC system, supporting structure, and attached hardware. The Reinforced Carbon-Carbon (RCC) system (including all RCC, supporting structure and attaching hardware) is an essential component of the Space Shuttle Orbiter Thermal Protection System (TPS) and has a Criticality Rating of 1 (loss of crew - loss of vehicle). The RCC composite consists of a reinforced carbon-carbon substrate that carries the structural loads, a tetraethyl orthosilicate impregnation that reduces inherent substrate porosity, a silicon carbide treatment that protects the substrate from oxidation, and a sealant coating that provides additional oxidation protection. These composite structures are attached to the shuttle by a metal support system. During initial manufacturing acceptance, the integrity of production composites used in the RCC system are checked at various points in production by physical tap, ultrasonic, radiographic, eddy current, weight gain, and visual tests. In addition, a flat plate control panel made in parallel with the production piece is destructively tested at various points in the production process. A projected design mission life has been established for each RCC component. These projections are based on analysis correlated to simulated flight load testing, and assume the presence of sound composite material and metal support structure. Visual external inspections and tactile checks are the only specified post flight inspections of $\mathrm{RCC}$ composite components. The planned interval for removing $\mathrm{RCC}$ composite components for more thorough inspection is typically several flights, unless their removal is dictated by an observed visual surface condition or necessitated by the requirement to provide access for other operations. Non-destructive testing methods like $\mathrm{X}$-ray have shown indications of RCC material defects not previously identified by visual inspection methods currently employed.

Prior to each Space Shuttle mission, the RCC panels undergo three inspections to ensure their integrity. The first is a post-flight, visual micro detail inspection of the TPS, which includes the RCC components. During this inspection, all exterior surfaces of the orbiter are closely examined and any damage is documented for repair. The 
second is a pre-rollout visual inspection of TPS that also includes the RCC components. This visual inspection checks again for any external damage. The third is a "tactile test",or hands-on test, that examines the hottest panels (panels 6-17) for evidence of loose or separated coating. These inspections are required and performed for every flight. During return-to-flight activities, all RCC panels undergo extensive non-destructive inspections (NDI) and non-destructive evaluations (NDE). NDI inspections include the use of thermography and CAT scans to detect imperfections or cracks in the structures on and below the surface. Thermography, a relatively new procedure at NASA Kennedy Space Center, uses high intensity light to heat areas of the panels. The panels are then immediately scanned with an infrared camera. As the panels cool, internal flaws are revealed. This form of NDI is in the development stage at KSC as RCC panel testing proceeds. Computer-aided CAT scans use magnetic resonance to scan the internal structure of the RCC panels. Panels are sent to a lab in Canoga Park, Calif., where a much larger machine is used to detect flaws. NDE methods include eddy current, ultrasound and X-ray. Eddy current is a technique that measures coating thickness and density properties of the panels. An electronic field detects disturbances in the panels, such as cracks and imperfections. During an ultrasound inspection, sound pulse waves are sent out to the component. As they are received back, defects and discontinuities are detected. X-rays of panels are performed at Lockheed Martin facilities in Dallas, Texas. In addition, several inspections of the metal components behind the RCC panels are performed. First, a visual inspection reveals any flaws to the naked eye. A dye-pen test, using a dye of red or purple, is applied to the component. Then, a blacklight is used to reveal any liquid that has penetrated the components, indicating cracks on or deeper than the surface. Despite all these inspections, thermal tiles fell off during the launch phase of Shuttle Columbia. In this paper, a micro flying robot fitted with suitable Raman spectrophotometers and lidars will be discussed to analyze hardware and materials for any type of fissures or cracks.

\section{RAMAN MICRO SPECTROPHOTOMETER}

Raman spectroscopy is a well-known analytical technique that is recently undergoing a tremendous revival due to technological advances in lasers, detectors and spectroscopic optical systems. When light is scattered by any form of matter, the energies of the majority of the photons are unchanged by the process, which is elastic or Rayleigh scattering. However, about one in one million photons or less, lose or gain energy that corresponds to the vibrational frequencies of the scattering molecules. This can be observed as additional peaks in the scattered light spectrum. The process is known as Raman scattering and the spectral peaks with lower and higher energy than the incident light are known as Stokes and anti-Stokes peaks respectively. Most routine Raman experiments use the red-shifted Stokes peaks only, because they are more intense at room temperatures. The availability of intense laser light sources from the 1970s onwards, and improved detector technology, with the increased Raman signal intensities resulting from these developments, started the techniques revival. Raman spectrometer hardware also started to upgrade, with the adoption of the multi-channel, silicon diode array and then the charge-coupled device (CCD) detectors developed for imaging technology. Prior to this, a point detector such as a photo-multiplier tube, was used to collect light from each point in the spectrum in turn, rotating or scanning the spectrograph, ${ }^{2} .^{3}$ This was a slow process, and collecting spectra took tens of minutes to several hours. However, the cooled CCD detectors now used can collect a wide spectrum almost instantaneously without scanning the spectrograph. The spectrograph is simply "parked" at the centre of the wave number region of interest and the CCD camera acquires the spectrum until good intensity is obtained, usually in a few seconds.

Another improvement to the raman systems, ten to twenty years ago is the addition of the holographic "notch filter" used to eliminate the Rayleigh scattering. Prior to the use of notch filters (or sometimes, interference edge filters), two successive spectrograph gratings were employed to filter the laser light from the Raman spectrum. Such so-called "triply dispersive" instruments, whilst allowing good precision and permitting acquisition of the Raman scattering from only a few wave numbers away from the excitation line, are complex and have a much lower throughput (sensitivity) than the single-grating systems. In contrast, the notch filter is inexpensive, easy to exchange when laser wavelengths are altered, and allows most of the Raman spectrum, to within about 50 $\mathrm{cm}^{-1}$ of the laser line or sometimes even less, to be collected. Such instruments are called singly dispersive. Raman spectra can now be acquired in real-time from almost any material in the world. It is a non-destructive and non-invasive method: samples inside glass bottles or transparent plastic containers such as drug packages can be analyzed without breaking the seal or risking contact with toxic or delicate samples. 


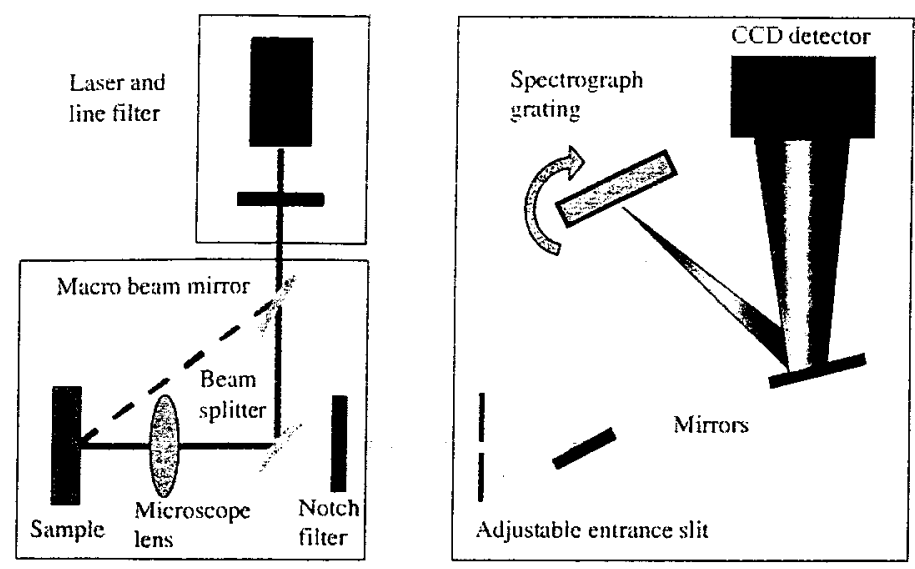

Figure 1. Raman Spectrometer Components.

There are two methods for analyzing the Raman spectrum of a sample: using a dispersive spectrograph with a diffraction grating or employing a Fourier-Transform (FT) spectrograph. The latter technique is often done using an accessory to a near-IR capable FT-IR spectrometer system, although to ensure adquate performance in the very near IR region used for FT-Raman, very high quality (gold coated) optics and InGaAs detectors are also required and the system becomes considerably more expensive than a standard FT-IR bench. Also, a rather powerful and expensive laser, typically a $1 \sim 5$ Watt CW YAG of $1064 \mathrm{~nm}$, must be used (in contrast, a dispersive Raman Spectrometer will operate with only a few percent of this power level). Raman scattering originates from molecular vibration modes in the material and produces spectral emission at a constant wave number shift from the exciting light, so a green laser of $532 \mathrm{~nm}$ should give a qualitatively identical spectrum to that obtained from $785 \mathrm{~nm}$ light. The main system hardware for raman spectrometers are the laser light source, the microscope sample chamber and the spectrograph or "polychromator". ${ }^{2}$

Light in a narrow, collimated beam from the excitation laser unit passes a line filter to remove any unwanted laser lines and sidebands (such as from pumping light in a diode laser, or a plasma lines in a gas laser), before passing via the beam splitter into the microscope. Additional optics including a macro beam mirror placed before the microscope, allowing macroscopic illumination of the Sample (dotted green line). Depending on the design of Raman Spectrometer, instruments may have more than one laser and laser selection optics. Confocal micro-Raman systems tend to minimize fluorescence signals because the objective lens focuses only on a small volume of material instead of the larger fluorescing volume actually excited by the laser in figure 2 . MacroRaman sampling acquires both the small Raman signal and much of the larger fluoresence background. Confocal micro-Raman can reduce fluoresence problems by virtue of the very small sample volume in the objective lens focus (green). The volume of sample producing fluorescence (red) is much greater but is largely ignored as the confocal aperture limits the range of focus contributing to the spectrum. In this figure 2 , only light from within the green in-focus region is analysed. The influence of fluorescence is greatly decreased.

Microscopic impurity particles can be detected even when embedded in a different (transparent) matrix because the different Raman signals are resolved by the microscope (spatially) and spectrometer (spectrally). For situations where the sample cannot be taken into the instrument, the excitation laser and scattered Raman signal can be fiber-optically coupled to the spectrometer. This scenario will be generally applicable to pre-launch inspection of the materials, tubes and other hardwares in the CEV. Probes allow powerful in-situ Raman measurements at a range of excitation wavelengths and with semi-micro capabilities using a long working distance lens and built-in colour CCD-TV camera for sample viewing. Alternative probes allow for immersion measurements in various liquid samples or in corrosive or other hazardous environments such as nuclear propellant tanks and other hazardous areas in the CEV. The Raman spectra and representative microscopic images can be used to characterize any type of contaminants or cracks. In the CEV, nano technology will play a crucial role in 


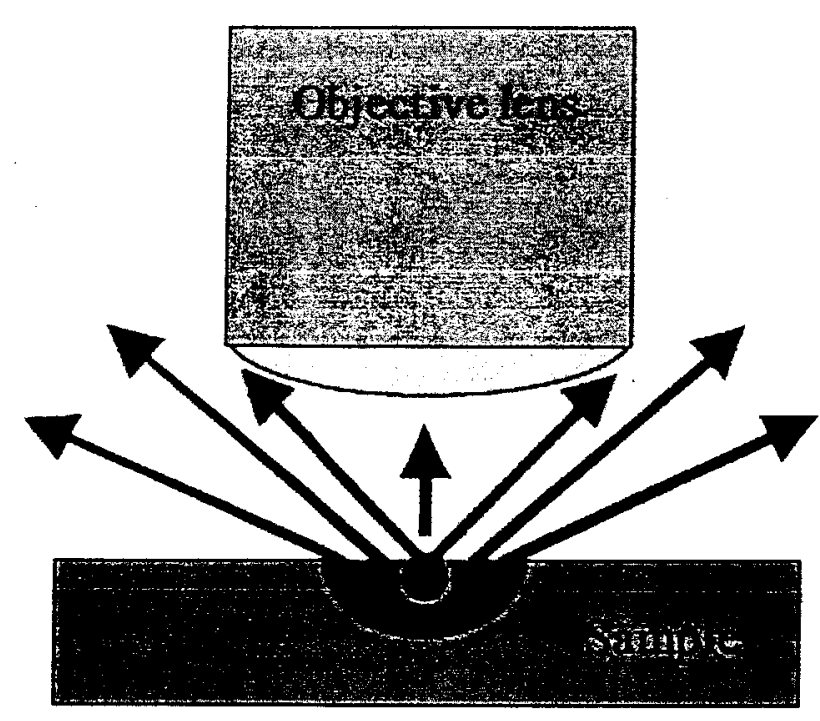

Figure 2. Reduction of fluoresence using confocal micro-raman spectrometer.

developing nanotubes. The primary method of analysis of nanostructures is Raman micro spectroscopy. The advantage of Raman spectroscopy is reliability, non-destructive and microscopic sample size. The micro Raman spectrophotometer will be mounted on a micro flying robot, which will keep constant monitoring of all hardware before launch of the CEV..

\section{MICRO FLYING ROBOT}

The miniaturization, extreme light weight and very low power consumption provide fundamental strategic challenges that accomplish future space exploration needs, which will have significant impact on mission cost, ${ }^{4},{ }^{5}, 6$ .$^{7}$ There are several research projects conducted around the world to miniaturise a flying robot to accomplish specific needs, ${ }^{8},{ }^{9},{ }^{10},{ }^{11},{ }^{12}{ }^{13}$ In Brussels, Alexander Van de Rostyne, together with leading suppliers of micro robotic components, recently developed a Pixelito in 2003, a 6.9 gram helicopter-like flying robot with full 4-axis control, similar to larger helicopters. ${ }^{14}$. Its two-bladed rotor has a diameter of $148 \mathrm{~mm}$ and can be controlled by an infra-red control device that enables the pilot to have full control over it in all the dimensions of space. In Oslo, Petter Muren, in close contact with the same team of component suppliers, developed the Proxflyer Micron, a 6.9 grams totally silent and aerodynamically stable coaxial rotor flying robot with a rotor diameter of $128 \mathrm{~mm}$. It is controlled via a 2 channel radio transmitter and an onboard FM radio reciever. The Airborne Microflyers developed by Georgia Tech. is a fixed wing model of the microflyer. The mechanical insect, known as an "Entomopter" is based around a new development called a Reciprocating Chemical Muscle (RCM) which is capable of generating automatic wing beating from a chemical energy source. ${ }^{15}$ MIT is developing Papa-TV-Bot, an autonomously hovering mobot with a wireless video camera. ${ }^{7}$ It can operate indoors and in obstacle rich areas where it avoids obstacles automatically.

Recently EPSON made a micro flying robot lighter by developing a new gyro-sensor that is a mere one fifth the weight of its predecessor, making the world's lightest gyro-sensor, ${ }^{16}{ }^{17}$ and is shown in figure 3 . Also helping to shed weight is the high-density mounting technology used to package the micro-robot's two micro controllers including EPSON original S1C33 family 32-bit RISC. Dynamic lift was boosted by thirty percent by introducing more powerful ultra-thin ultrasonic motors and newly designed optimally shaped main rotors. EPSON added an image sensor unit that can capture and transmit aerial images via a Blue tooth wireless connection to a monitor or ground station and they also devised two LED lamps that can be controlled as a means of signaling. 


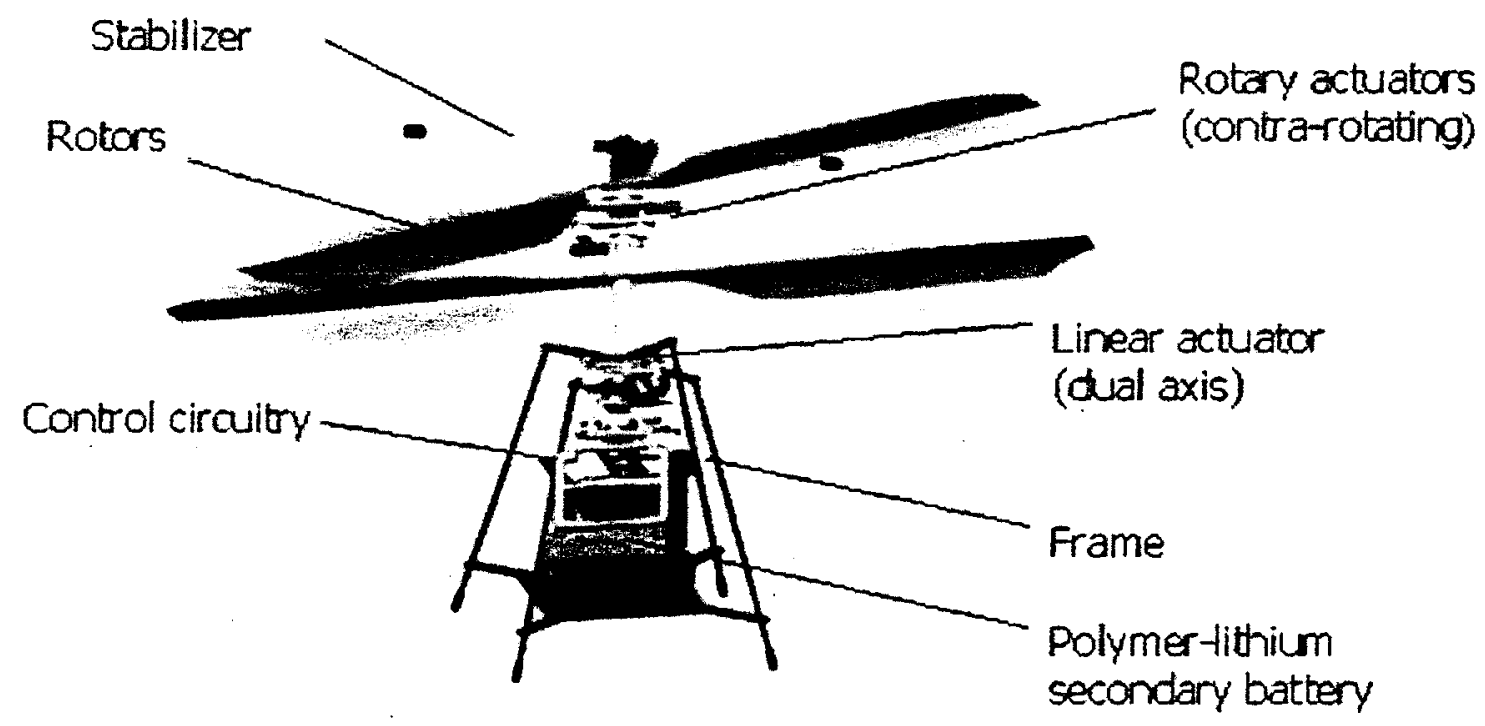

Figure 3. EPSON's micro flying robot.

The specific characteristics of EPSON's micro flying robot are

- EPSON's own gyro-sensor (world's smallest and lightest).

- Two EPSON original SIC33 family of 32-bit RISC microprocessors, on board with high-density mounting.

- Ablility to use commercially available battery (polymer-lithium battery light weight and more efficient drive circuitry).

- Actuator with the world's highest power-weight ratio, providing 30 percent more lift than the earlier version of $\mu F R$.

- World's first linear actuator-equipped balance control system for higher precision and faster response.

The EPSON'S gyro sensor is based on a monocrystalline quartz crystal. By leveraging EPSON's original crystal microprocessing technology, low-power analog circuit technology, and packaging technology, the team is able to achieve a gyro sensor measuring just $5.0 \times 3.2 \times 1.3 \mathrm{~mm}$, making it the world's smallest gyro sensor with built-in drive circuitry. ${ }^{17}$ This subminiature angular velocity sensor is ideally designed for use in systems that correct camera vibrations. The sensor will enable compact digital cameras, camera-phones, and other products to be equipped with a high-performance motion correction feature.

\section{General Specifications}

- Power: $4.2 \mathrm{~V}$

- Power consumption: $3.5 \mathrm{~W}$

- Dimensions: Diameter: $136 \mathrm{~mm}$, Height $85 \mathrm{~mm}$

- Maximum lift: $17 \mathrm{~g} / \mathrm{f}$

- Weight: $12.3^{\circ} \mathrm{g}$

- Flight time: 3 minutes 


\section{TECHNICAL CHALLENGES OF MICRO FLYING ROBOT}

Although NASA develops exceptionally reliable space systems, all materials, structures, and electronics eventually will fail. This is often the result of a slow fault progression over a long period of time. Material imperfections and weaknesses (e.g., cracks, fatigue, etc.) can begin early in the operational life of a component, often at microscopic levels. As working loads (e.g., thermal, vibration, acceleration, etc.) continue to stress the material, incipient faults appear. Eventually, damage patterns develop with overt manifestations that can be identified with signal detection and fault isolation technology. Micro flying robots with micro Raman spectrophotometer could provide a prognostic framework by which incipient faults can be detected before they progress to the point of failure, triggering condition-based maintenance actions or fault accommodation/recovery mechanisms. Design of transportation systems for maintainability by crews during flight (or during surface operations) should be an overarching design and engineering principle for the Crew Exploration Vehicle.

There are several key technical challenges for present state of micro flying robot which can be accommodated in future NASA's space mission. They are as follows:

Aerodynamic characteristics of micro flying robot, which includes lift, drag, payload design, increasing sustainable flying time (at least 30 minutes instead of 3 minutes). Present design has to be modified for long duration flight. Present micro flying robots operate indoor and need to be redesigned to fly in micro gravity and harsh environments.

Present power supply system sustains 3 minutes of flying time. Onboard battery design has to be enhanced in such a way that it can supply power for long duration flight. The battery recharging mechanism has to be developed since there is no recharging mechanism of on-board batteries in its present state. The battery charger can be designed like a mobile base station (similar to military tank), where the micro flying robot can land on the charger. The battery charger can be considered a base station, where it can hold micro Raman spectrophotometers, computers and other ancillary devices. Even the base station can be another robot or an instrument or Crew Exploration Vehicle or astronaut suit.

Micro Flying Robot controls : Existing microflyers are controlled by humans or tele-operated. Autonomous on-board controls have to be designed in such a way that there is an autonomous take-off, traversing, recharging of power, stable flight, and smooth landing. The controls have to be designed to withstand harsh environments and micro gravity.

Communication Systems : Present communication system are based on Blue-tooth wireless technology. In a harsh environment, the system will have more interference with the base station. Evolvable antennas and necessary communication system have to be addressed to overcome communication problems.

Sensors: Micro flying robot's weight and size is a key factor with respect to the payload of the Crew Exploration Vehicle. The micro/nano sensors are suitably designed to accomplish mission requirements with the least amount of energy expenditure. A plug-in style sensor mating technique to the micro flying robot may need to be developed.

Instrumentation : Ancillary instrumentation for power storage, generation, specific needs of sensors, sensor holding and discharging instruments should be developed.

Solving the above technical challenges will enable EPSON's micro flying robot to satisfy minimum requirements of specific mission challenges. Distributed collaborative micro flying robots (flock of robots) to gather data, images and samples will achieve future space mission needs.

\section{CONCLUSION}

Micro flying robot technology can be adopted to address a given mission needs from pre-launch inspections to on-board inspection and health monitoring of a CEV. The micro flying robot combined with micro raman spectrophotometer probes, a vertical scanning of the materials and hardware can be monitored for any stress related cracks or fissures. Micro flying robot can also be assistant robots for a primary robot using a master and 
slave approach. The primary robot can control all instrumentation of the micro flying robot. The primary robot can act as a planner and scheduler for the micro flying robot. The micro flying robot can be used during human missions as a robotic assistance. Since micro flying robot development is at the initial stages, new innovative concepts are needed to achieve NASA's mission needs and goals. The micro flying robot can be used by human missions as a robotic assistance. Apart from NASA missions, micro flying robots can be used as a security surveillance tool, as an unmanned aerial vehicle for analyzing enemy territory.

\section{ACKNOWLEDGMENTS}

We would like to acknowledge useful comments, suggestions and discussions from Dawn Clements and Matthew Dortenzio. The authors wish to thank Dr. Michael Shafto and Dr. Butler Hine for their financial support through the CICT program at NASA Ames Research Center.

\section{REFERENCES}

1. NASA, "http://www.nasa.gov," 2005.

2. JASCO, "http://www.jascoinc.com/pages/history.html," 2004.

3. JASCO, "http://www.jascoinc.com/literature/pdf/appnotes/," 2004.

4. M. S. Francis, "http://www.darpa.mil/tto/mav/," 1997.

5. O. H. Richard and C. Eugene, "Future technology-driven revolutions in military operations," 1992.

6. A. Datta, "http://www.enae.umd.edu/agrc/design00/marv.html," 2000.

7. S. Marti, "http://web.media.mit.edu/ stefanm/ffmp/ffmp.html," 1998.

8. W. R. Davis, "Micro-uav," in 23rd Annual AUVSI symposium, 1996.

9. R. Fearing, "Wing transmission for a micromechanical flying insect," in IEEE ICRA, 2000.

10. R. A. F. Jr. in Advanced automation for space missions, NASA Scientific and Technical Information Branch, 1982.

11. J. Zufferey, D. Floreano, M. V. Leeuwen, and T. Merenda, "Evolving vision-based flying robots," in Proceedings of the second International workshop on Biologically motivated computer vision, LNCS 2525, 2002.

12. M. Rais-Rohani and G. Hicks, "Multidisciplinary design and prototype development of a very small remotelypiloted reconnaissance airplane," in SAE/AIAA World Aviation Congress and Exposition, 1997.

13. M. Rais-Rohani and G. Hicks, "Multidisciplinary design and prototype development of a micro air vehicle," Journal of Aircraft 36, No 1, 1999.

14. P. Muren, "http://www.proxflyer.com/," 2004.

15. R. Michelson, "http://gtresearchnews.gatech.edu/reshor/," 1998.

16. EPSON, "http://www.epson.co.jp/e/newsroom/," 2004.

17. EPSON, "http://www.alcom.nl/images/nieuws/," 2004. 